\title{
Metasurface-based Optical Liquid Crystal Cell as an Ultra-Thin Spatial Phase Modulator for THz Applications
}

Oleksandr Buchnev*, Nina Podoliak, Korbinian Kaltenecker, Markus Walther, and Vassili A. Fedotov

Dr. O. Buchnev

Optoelectronics Research Centre and Centre for Photonic Metamaterials, University of Southampton, SO17 1BJ, UK

E-mail: O.Buchnev@soton.ac.uk

Dr. N. Podoliak

Mathematical Sciences, University of Southampton, SO17 1BJ, UK

Dr. K. Kaltenecker

Faculty of Physics and Center for NanoScience, Ludwig-Maximilians-Universität München, 80799, Munich, Germany

Department of Molecular and Optical Physics, University of Freiburg, D-79104, Germany

Dr. M. Walther

Department of Molecular and Optical Physics, University of Freiburg, D-79104, Germany

Dr. V. A. Fedotov

Optoelectronics Research Centre and Centre for Photonic Metamaterials, University of Southampton, SO17 1BJ, UK

ABSTRACT: A concept of an efficient ultra-thin spatial phase modulator for correcting terahertz wavefronts in transmission with sub-wavelength spatial resolution is experimentally demonstrated. It exploits a combination of spatially addressable resonant planar metamaterials (so-called metasurfaces) and an optically thin $(<0.04 \lambda)$ low-voltage $(<20 \mathrm{~V})$ liquid crystal cell. The device allows 'imprinting' 2D phase profile of any spatial complexity with a spatial resolution better than $0.23 \lambda$, and can be readily fabricated using the well-established LC-device technology and highresolution photolithography technique.

KEYWORDS: terahertz, spatial light modulators, liquid crystal devices, planar metamaterials, hybrid metamaterials 


\section{INTRODUCTION}

Spatial light modulators (SLMs) are optical devices designed to manipulate the wavefronts of light beams in space and time. Among the available technologies that are currently widely used are SLMs based on liquid crystals (LCs) - highly birefringent materials with the optical properties that can be effectively controlled by electric field or light. ${ }^{1}$ In the simplest configuration, an LC-based SLM is an array of spatially addressable LC pixels (similar to those found in LC displays), which introduce programmable retardation to the local phase of reflected or transmitted light wavefronts via electrically-induced re-orientation of LC molecules. ${ }^{2,3}$ The application of SLMs in optics and photonics ranges from beam steering to wavefront correction and spatial structuring of visible and infrared beams, as well as image processing. ${ }^{4-6}$

SLMs are also essential for many $\mathrm{THz}$ applications. In particular, they would enable the optimization of the spatial resolution and illumination uniformity in THz-imaging systems employed in biotechnology, material testing, health, safety and security areas. ${ }^{7}$ Also, correcting the wavefronts of $\mathrm{THz}$ pulses is needed for the control of the far-field intensity distribution of timedomain spectrometers, including the optimization of $\mathrm{THz}$ sources and beam focalization of scanning THz time-domain imaging systems. ${ }^{8}$ While the phase shifts required for wavefront correction often do not exceed $\pi / 2$, the development of efficient SLMs for THz radiation remains one of the main challenges of the technology. ${ }^{9}$ Even the operational principle of LC-based SLMs (despite being efficient and straightforward) is difficult to directly transfer into the $\mathrm{THz}$ domain. Indeed, although LCs behave well in the $\mathrm{THz}$ frequency range exhibiting a wide transparency window, due to relatively low birefringence of the existing LCs efficient $\mathrm{THz}$ phase modulation is possible only with LC layers exceeding several hundred micrometers in thickness. Correspondingly, robust alignment and spatial control of LCs in such thick optical cells with an 
externally applied electric field requires very high driving voltage, generally in excess of $100 \mathrm{~V}^{10}$ and, hence, is a considerable technological challenge. The existing alternative approaches involve the use of photonic crystals ${ }^{11,12}$, graphene ${ }^{13,14}$, phase-change oxides and semiconductors ${ }^{15-17}$, and suffer from a number of drawbacks as well, such as small active area and challenging fabrication, while some require cryogenic cooling. ${ }^{15,16}$

We argue that the operational principle of LC-based SLMs can be readily extended on the spatial modulation of terahertz wavefronts with the use of metamaterial technology. Metamaterials have revolutionized the way electromagnetic waves can be manipulated and controlled, ${ }^{18}$ and enabled a substantial improvement in the performance of terahertz devices. ${ }^{19-23}$ In particular, efficient modulation of $\mathrm{THz}$ radiation was achieved by hybridizing metamaterials with chalcogenides, ${ }^{24,25}$ phase change metal oxides, ${ }^{26,27}$ semiconductors, ${ }^{28,29}$ micro electro-mechanical systems ${ }^{30,31}$ and graphene. ${ }^{32,33}$ LC hybridized metamaterials have contributed significantly to the development of effective modulators, ${ }^{34,35}$ tunable filters, ${ }^{36,37}$ switchers, ${ }^{38,39}$ and SLMs ${ }^{40,41}$ for a wide spectral range from visible to $\mathrm{THz}$ frequencies. A particularly efficient electrically-driven mechanism of amplitude and phase modulation of $\mathrm{THz}$ radiation has been demonstrated in transmission with a thin low-voltage $\mathrm{LC}$ cell combined with a resonant $2 \mathrm{D}$ metamaterial $^{42}$ and, later, 3D metamaterial. ${ }^{43}$ Also, a programmable $\mathrm{THz}$ beam steering metasurface operating in reflectance and employing binary encoding of its phase profile was shown recently by Wu et.al. ${ }^{41}$ In this paper, we propose the concept of an optically thin large-area spatial phase modulator (SPM) with subwavelength pixel resolution and continuously controlled phase retardation for correcting aberrations of terahertz waterfronts in transmission. Importantly, our SPM operates without diffraction, i.e. it does not produce diffraction orders other than the $0^{\text {th }}$ order, and therefore can be used to correct THz wavefronts directly. It is based on a low-voltage $(<20 \mathrm{~V})$ electro-optical LC cell hybridized with spatially addressable metamaterial stack, which can be placed in the path of 
terahertz radiation. Below we describe the design and operating principle of such a modulator, and investigate its operational range and functional capability for correcting $\mathrm{THz}$ wavefronts using numerical simulations. We also fabricated a prototype of the SPM and offer an experimental proofof-concept demonstration by configuring the device to tilt transmitted wavefronts at $0.8 \mathrm{THz}$.

\section{DESIGN AND OPERATING PRINCIPLE OF THZ SPATIAL PHASE MODULATOR}

The conceptual design of the proposed terahertz spatial phase modulator is presented in Figure 1a. It constitutes a hybrid optically thin LC cell composed of two identical planar metamaterials (metasurfaces) supported by transparent dielectric substrates and a layer of a nematic LC sandwiched between them.

(a)

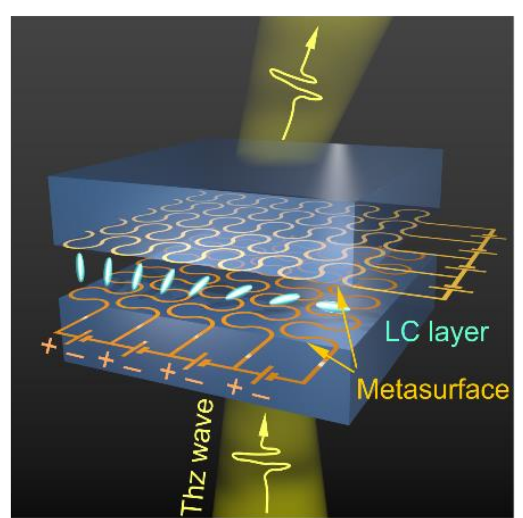

(c)

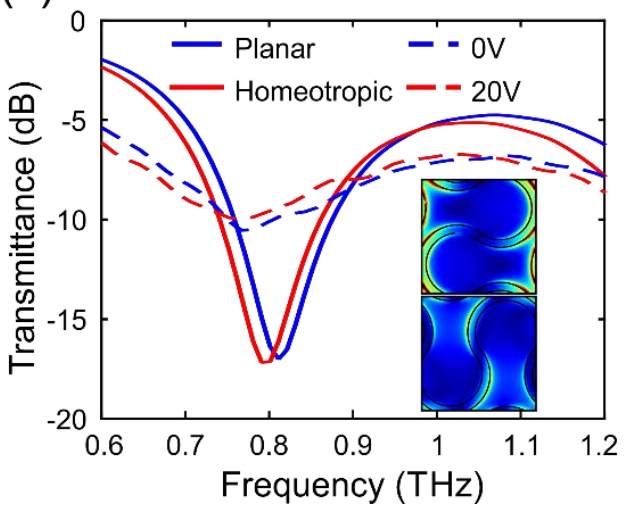

(b)

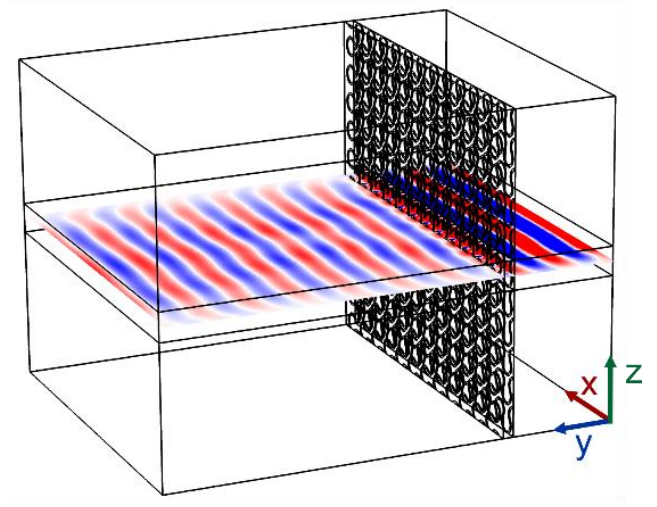

(d)

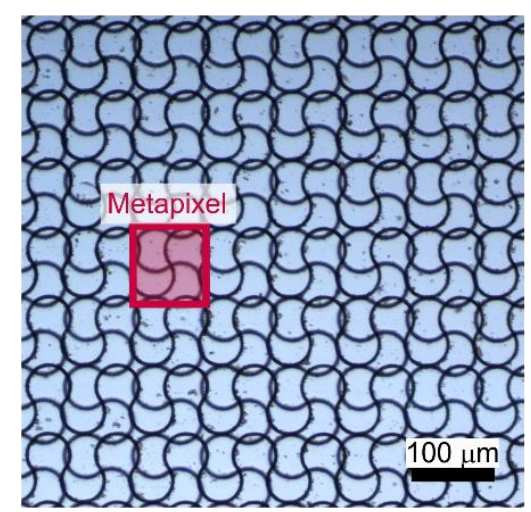


Figure 1. (a) An artistic impression of a spatial phase modulator. (b) The geometry of the COMSOL model used for simulating the operation of the spatial phase modulator. (c) Simulated (solid lines) and measured (dashed lines) transmittance spectra of the spatial phase modulator presented for two limiting cases of LC uniform alignment corresponding to planar/ $0 \mathrm{~V}$ (blue) and homeotropic/ $20 \mathrm{~V}$ (red). Phase dispersions corresponding to the transmission spectra are shown in Figure S1 in Supporting Information. Inset shows simulated distributions of the amplitude of the local electric field induced in the bottom and top metamaterial units by horizontally polarized wave at a resonant frequency of $0.8 \mathrm{THz}$. (d) An optical microscope image of the fabricated spatial phase modulator designed to operate at a frequency of $0.8 \mathrm{THz}$.

The metamaterial pattern is anisotropic featuring a periodic array of meandering wires, which are formed by interconnected sub-wavelength metallic split rings (circular arcs with the central angle of $250^{\circ}$ ); the elementary unit of the pattern (metamaterial unit or metamolecule) is a square containing two metallic split rings. Such a pattern renders the metasurfaces as sets of spatially addressable electrodes, where an electric potential can be applied to any meandering wire independently. When the patterns of the metasurfaces are rotated by $90^{\circ}$ with respect to each other (as shown in Figure 1a), a potential difference can be selectively applied between any two overlapping metamaterial units from the top and bottom metasurfaces. Such a twisted arrangement also makes the transmission response of the metamaterial stack insensitive to the polarization of incident light.

If initially the molecules in the LC layer are all oriented unidirectionally and parallel to the plane of the cell (so-called planar LC alignment), the application of a potential difference will perturb the orientation of LC molecules between the selected metamaterial units, forcing LC molecules there to align perpendicular to the plane of the cell (so-called homeotropic LC alignment). In turn, that will locally affect the refractive index and, more importantly, shift the resonance frequency of the selected metamaterial units introducing an additional change in the phase of the waves they scatter (i.e., optical delay). ${ }^{42}$ Since the change of the refractive index is controlled by the tilt of LC molecules, it can be tuned continuously by varying the magnitude of locally applied voltage, as 
schematically illustrated in Figure 1a. We note that the resulting spatially addressable hybrid cell mimics an LC display based on the passive matrix scheme, where every pixel in the matrix (a pair of overlapping metamaterial units in our case) can be activated individually, by selecting an appropriate column and row of the matrix (metamaterial stack in our case). Given sub-wavelength dimensions of the addressable metamaterial units (metapixels), the proposed concept of a spatial phase modulator does not engage diffraction into the $1^{\text {st }}$ (or any higher) orders, and enables one to modify or correct the wavefront of a terahertz beam directly, by placing the modulator in the path of the beam.

Below we illustrate the operating principle of our SPM, first, by modelling in $\mathrm{COMSOL}^{44}$ the propagation of a terahertz plane wave through the modulator, which is configured to tilt the transmitted wavefronts. The geometry of the model is presented in Figure 1b. It featured a rectangular computational domain, which encompassed a pair of metasurfaces comprising 12 x 12 metamolecules each. All sides of the domain were defined as scattering boundaries effectively rendering the domain infinitely large. The incident beam has a supergaussian profile with the diameter of $0.85 \mathrm{~mm}$, was polarized along $x$-axis and propagated along $y$-axis from right to left. The composition of the modelled structure and its dimensions were defined as follows. The substrates of the metasurfaces were assumed to be semi-infinite slabs of fused quartz $\left(n_{\mathrm{s}}=2.1^{45}\right)$ facing outwards. The LC layer between the metasurfaces had a thickness of only $12 \mu \mathrm{m}$ and was characterised by an ordinary, $n_{\mathrm{o}}$, and extraordinary, $n_{\mathrm{e}}$, refractive indices of $1.554+0.15 i$ and $1.941+0.15 i$, respectively, found in a highly birefringent nematic LC mixture $1825 .{ }^{46}$ The wires of the metamaterial pattern had a width of $6.0 \mu \mathrm{m}$, and were modelled as perfect electric conductor surfaces. The period of the pattern (i.e., the size of the metamaterial units) was set to $85 \mu \mathrm{m}$, which prevented the metamaterial array from scattering normally incident waves into the $1^{\text {st }}$ (or higher) order diffraction modes in both the substrate and air at frequencies below 1.7 THz. Overall, the 
simulated structure had an area of $\sim 1 \mathrm{~mm}^{2}$ and, therefore, it was large enough to serve as a realsize model of the proposed SPM.

We begin by characterizing the transmission response of the SPM in the frequency range $0.6-1.2 \mathrm{THz}$ for two cases of spatially uniform LC alignment, namely planar alignment (LC molecules are parallel to $x$-axis) and homeotropic alignment (LC molecules are parallel to $y$-axis) prescribed for all metapixels of the SPM. Figure 1c shows that in both cases the transmission is resonant at around $0.8 \mathrm{THz}$ but the resonance shifts by about $15 \mathrm{GHz}$ when $\mathrm{LC}$ alignment is switched between planar and homeotropic states. As noted above, the shift of the resonance results from a change of the refractive index of the LC layer $\left(n_{\mathrm{e}}\right.$ in the case of planar alignment, and $n_{\mathrm{o}}$ in the case of homeotropic alignment) and should be accompanied by a substantial change of the resonant electromagnetic phase near $0.8 \mathrm{THz} .{ }^{42}$ The nature of the resonance was previously discussed in reference. ${ }^{47}$ Briefly, the resonance signifies the excitation of standing waves of electrical currents in the pattern of the metasurface when the length of the wire accommodated by one unit of the pattern becomes equal to a half of the wavelength of incident radiation in the surrounding dielectric medium. It is driven by the electric-field component of the incident wave parallel to the length of the meandering wire. Despite the anisotropy of the pattern, the mutually orthogonal orientation of the metasurfaces within the metamaterial stack ensures that at least one of them is at resonance with the incident wave regardless of its polarization. In particular, in our case, the resonance is exhibited by the first metasurface facing the incident wave, as evident from the inset to Figure 1c. Importantly, due to high localization of electromagnetic fields around the metamaterial pattern at the resonance, the LC layer in our device could be made as thin as that of a standard LC cell found in many optical LC devices.

To establish the operation limits of our SPM, we consider the maximum change in the retardation of the transmitted wave that can be induced by each metapixel upon LC transition from planar to 
homeotropic state. Figure 2a shows how the induced phase change (red curve) varies with the tilt angle of LC molecules, $\theta$ - the parameter that describes the alignment of the LC layer and, in practice, can be gradually tuned by the voltage applied across an activated metapixel. One can see that the phase change is naturally zero at $\theta=0^{\circ}$ (planar alignment) but gradually increases with increasing $\theta$, reaching a maximum of $32^{\circ}$ at $\theta=90^{\circ}$ (homeotropic alignment). Importantly, such a large phase change is observed at $0.8 \mathrm{THz}$ and results almost exclusively from tuning the resonant response of the metasurfaces. We confirmed this by calculating the phase change induced by the LC layer alone, i.e. in the absence of the metasurfaces (see blue cure in Figure 2a). Clearly, the phase change does not exceed $4.5^{\circ}$, which can be readily appreciated given that the thickness of the LC layer was only $12 \mu \mathrm{m}$.

(a)

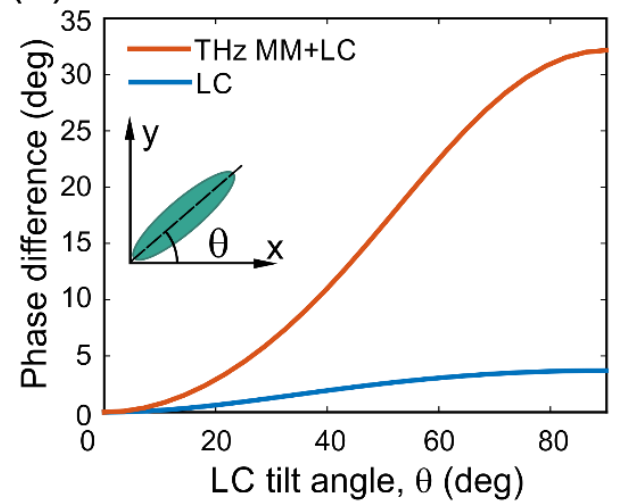

(c)

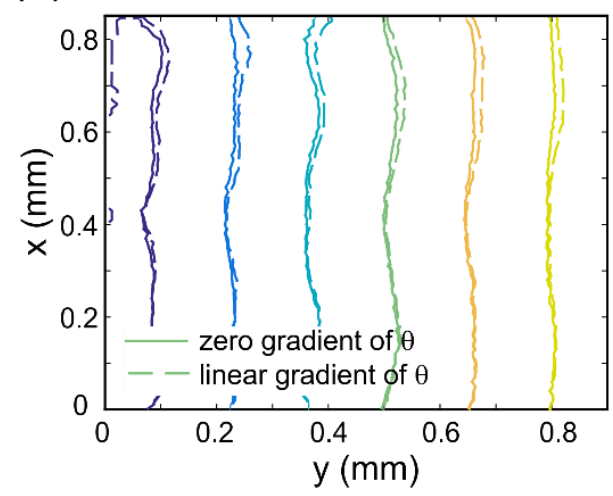

(b)

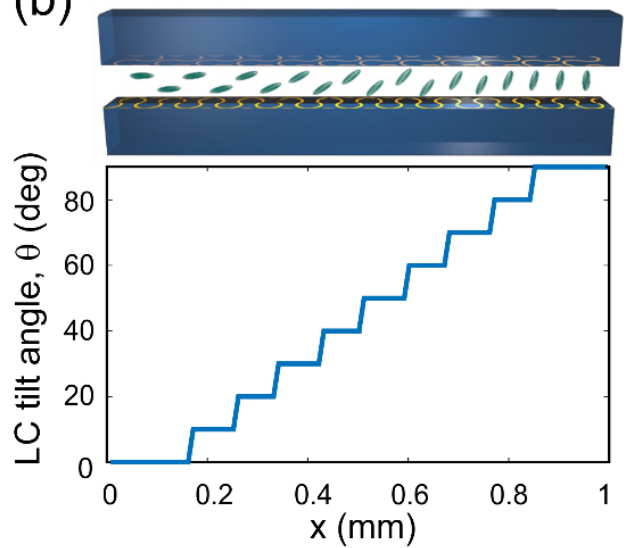

(d)

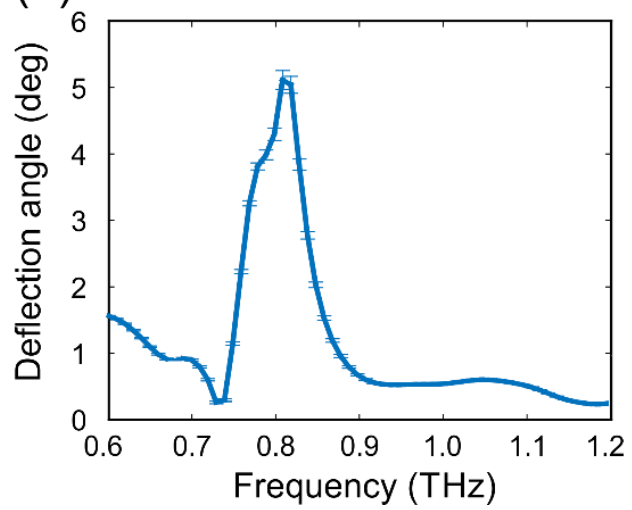


Figure 2. (a) Phase retardations induced by the spatial phase modulator (red) and LC layer alone (blue) at $0.8 \mathrm{THz}$ as functions of LC tilt angle, $\theta$ (red). Inset explains how $\theta$ is defined. (b) Spatial variation of the LC tilt angle along the $x$-axis assumed in the simulations. Inset shows schematically the orientation of molecules in the LC layer that corresponds to the spatial variation of the LC tilt angle below. (c) Simulated contours of the wavefronts transmitted by the spatial phase modulator at $0.8 \mathrm{THz}$ with (dashed lines) and without (solid lines) the gradient of LC alignment introduced along the $x$-axis. The contours are separated by a phase difference of $2 \mathrm{rad}$. (d) Calculated beam deflection produced by the spatial phase modulator due to the gradient of LC alignment as a function of frequency.

Finally, we configure our SPM to deflect transmitted wavefronts by allowing the tilt of the molecules within the LC layer to increase linearly from metapixel to metapixel along $x$-axis (see inset to Figure 2b). Such a configuration renders the SPM effectively as a very thin prism, which deflects the transmitted wavefronts directly, without producing the $1^{\text {st }}$ and higher diffraction orders. We note that in practice, the potential difference applied to metapixels will vary discretely, and to respect that in our model we kept the tilt angle constant within each metapixel, which resulted in a stairway-like profile of $\theta(x)$, as shown in Figure 2b. The effect of such an SPM configuration on the propagation of a plane wave at $0.8 \mathrm{THz}$ is demonstrated in Figure 2c, where we compare the simulated contours of the waterfronts transmitted by the SPM with and without the gradient of $\theta$. When the gradient of $\theta$ is present the wavefront undergoes a noticeable deflection towards y-axis. We define the corresponding deflection angle as the angle of rotation of the wavefronts that they acquire locally relative to their initial orientation, i.e. in the absence of the gradient. To minimize the error due to limited accuracy of our simulations and finite spatial resolution of the wavefront mapping, we calculated the angle of the wavefront rotation at every point of the map and then averaged it over the mapped area. The result we obtained for the deflection angle is $2.4^{\circ}$. We recall that in our model the wave propagates in a fused quartz (with the refractive index of 2.1) and, as dictated by Snell's law, the angle of deflection will increase to $5^{\circ}$ as soon the wave leaves the substrate. Figure 2d shows the spectral dependence of the average deflection angle of the 
transmitted wavefronts in air, which was extracted directly from the wavefront maps corresponding to different frequencies. The error bars reflect the uncertainty in extracting the deflection angle due to distortions of the transmitted wavefronts. Our data confirm that the efficiency of phase modulation reaches a maximum at the metamaterial resonance and, thus, emphasize the key role of the metasurfaces as phase control elements in the proposed concept of a terahertz spatial phase modulator. The wavefront tilt angle can be increased by making the gradient of LC alignment across the SPM steeper, or by employing LC materials with higher birefringence. ${ }^{48,49}$ For instance, using recently developed LC mixtures with birefringence of $0.75^{48}$ we could obtain the maximal phase delay in a single metapixel of $55^{\circ}$. This would enable our SPM to tilt the transmitted $\mathrm{THz}$ wavefronts by up to $9^{\circ}$. Also, the characteristics of the SPM can be improved further by introducing an additional resonant element into the pattern of the metamaterial unit. Such an approach enables one to replace a conventional metamaterial resonance with a Fano resonance, which is characterised by stronger phase dispersion and higher levels of transmission (currently $-10 \mathrm{~dB}){ }^{50}$ While the suggested measures will undoubtedly improve the efficiency of our SPM as a beam deflector, we emphasize that wavefront tilting serves merely as a demonstration of the operational principle and robustness of the proposed concept. Indeed, it requires "imprinting" a simple phase profile corresponding to a linear phase gradient and is, therefore, very easy to verify experimentally and compare with the prediction of numerical modelling, as we have done below.

\section{EXPERIMENTAL DEMONSTRATION OF THZ SPATIAL PHASE MODULATOR}

We demonstrate the robustness of our SPM concept in an experimental study, where we imaged the propagation of terahertz waves directly, by measuring the electric field of the waves along $x$ and $y$-axes. For this demonstration we fabricated a structure, which resembled very closely the 
spatially addressable hybrid metamaterial-LC cell that we modelled in the previous section (Figure 1d). It featured two metasurfaces fabricated with the help of high-resolution photolithography from $200 \mathrm{~nm}$ thick copper films, which were spattered on $1 \mathrm{~mm}$ thick quartz substrates (coated beforehand by a $5 \mathrm{~nm}$ thick chromium layer to facilitate copper adhesion). Every wire of the metamaterial pattern was connected to a separate $0.5 \times 1.5 \mathrm{~mm}^{2}$ copper patch acting as an external miniature contact pad. The area of each of the fabricated metasurfaces was $5 \times 5 \mathrm{~mm}^{2}$. The hybrid cell was assembled by placing one metasurface above another with their wire patterns facing each other. As in the modelled structure, the patterns were rotated with respect to each other by $90^{\circ}$ and carefully aligned to ensure the complete overlap between the metamolecules of the top and bottom metasurfaces (see Figure 1d). The gap between the metasurfaces was maintained by $12 \mu \mathrm{m}$ large silica spacers placed there during assembly. The assembled cell was filled with a highly birefringent nematic LC mixture $1825^{42}$ via capillary action. Prior to assembly a thin layer of polyimide was spin-coated over the wire patterns of the top and bottom metasurfaces and uniformly rubbed with a cloth, respectively, along and across the length of the meandering wires. That ensured uniform planar alignment of LC once it had filled the gap, which we confirmed by inspecting the cell under polarized microscope. A photograph of the assembled SPM sample is presented in Figure S2 in Supporting Information.

The transmission of the assembled hybrid cell was first characterized in planar and homeotropic states, which corresponded to $0 \mathrm{~V}$ and $20 \mathrm{~V}$ applied uniformly over the cell, respectively. The voltage was supplied by connecting all contact pads of the top metasurface to an AC signal generator (producing square waveform profile with a frequency of $1 \mathrm{kHz}$ ) while maintaining the contact pads of the bottom metasurface all connected to ground. The transmission measurements were performed at normal incidence using a conventional terahertz time-domain spectroscopy setup based on photoconductive antennas optically gated by the output of a mode-locked 
Ti:sapphire laser (<20 fs, $800 \mathrm{~nm}, 75 \mathrm{MHz}$ repetition rate). The setup produced a linearly polarized terahertz beam, which was focused onto the cell to a spot with a diameter of $1 \mathrm{~mm}$. The acquired transmission spectra are shown in Figure 1c. They correlate reasonably well with the spectra calculated numerically, exhibiting a resonance near $0.8 \mathrm{THz}$ which appears to red-shift by $15 \mathrm{GHz}$ when the state of the cell changes from planar to homeotropic. The broadening of the resonance and somewhat higher transmission at its center are attributed to an increase of Ohmic resistance in sputtered copper, as well as to non-zero dissipative losses in LC 1825. The response time of the demonstrated SPM is $75 \mathrm{~ms}$. It was determined as a sum of the switching on and off time of liquid crystal in the SPM upon applying $20 \mathrm{~V}$. The on and off times were measured optically with a standard set-up for liquid crystal research by monitoring the intensity of a laser beam passed through the SPM placed between crossed polarizers. The on time was determined as the time interval during which the cross polarized intensity decayed to 1/e of maximum level, and the off time was given by the increase of the intensity to $1 / \mathrm{e}$ of saturation level. ${ }^{51,52}$ While the response of our SPM is slower than that reported for some of THz metamaterial systems loaded with liquid crystals, ${ }^{53,54}$ we note that shorter switching times are not critical for the operation of SPMs (i.e. eliminating aberrations of $\mathrm{THz}$ wavefronts) and, if needed, can be achieved by using dualfrequency liquid crystals. ${ }^{54}$

Our SPM was configured to tilt transmitted wavefronts by connecting sequentially, via identical resistors $(1 \mathrm{k} \Omega)$, ten contact pads of the top metasurface. The first contact pad in the resulting chain was then attached to the output of the voltage generator set to $20 \mathrm{~V}$, while the last contact pad, as well as all contact pads of the bottom metasurface, were connected to ground. Such a configuration ensured that the applied voltage would increase linearly from 0 to $20 \mathrm{~V}$ along a ten-pixel wide strip, which corresponded to the section of the SPM illuminated by the terahertz beam. The wavefronts transmitted by the configured section of SPM were visualized using a state-of-the-art THz-field 
imaging technique. It is described in detail in reference, ${ }^{55}$ but for the benefit of the reader we provide a schematic of the system in Figure $\mathbf{S 3}$ in Supporting Information. In particular, in the 0.6-1.2 THz range of frequencies it enabled the mapping of both the phase and amplitude of the electric-field component of propagating waves along the direction parallel ( $x$-axis) and perpendicular ( $y$-axis) to the face of the SPM with spatial resolutions of, respectively, $7.5 \mu \mathrm{m}$ and $15 \mu \mathrm{m}$, and with a frequency resolution of $0.05 \mathrm{THz}$.

Figure 3a and $\mathbf{3 b}$ compare the actual wavefronts transmitted by the SPM at the metamaterial resonance for two limiting cases, namely $0 \mathrm{~V}$ and $20 \mathrm{~V}$ gradients applied to the configured section of the SPM. Although the wavefronts appear somewhat curved (as a result of tight focusing of the incident beam realized in the THz setup), they can be seen to tilt towards $y$-axis when the voltage gradient is present. The tilt is better visible in Figure 3c, where we superimposed the contours of the corresponding wavefronts. We calculated the angle of the wavefront rotation at every point of the map and then averaged it over the mapped area (see Supporting information for details). The result is plotted in Figure 3d as a function of the frequency of an incident wave. The wavefront deflection angle is seen to reach a maximum of $4.5^{\circ}$ at $0.8 \mathrm{THz}$, while remaining below $1^{\circ}$ at other frequencies in the range 0.6-1.2 THz. Clearly, the experimentally measured dispersion of the wavefront deflection angle is in a very good agreement with the prediction yielded by our numerical model (compare Figure 3d and 2d). 
(a)

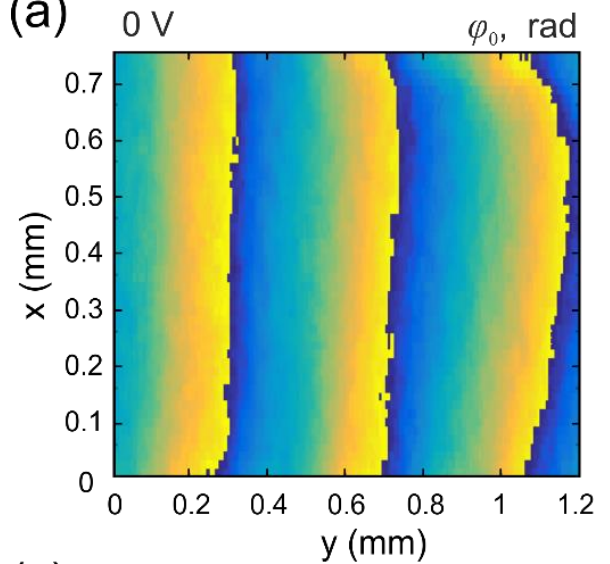

(c)

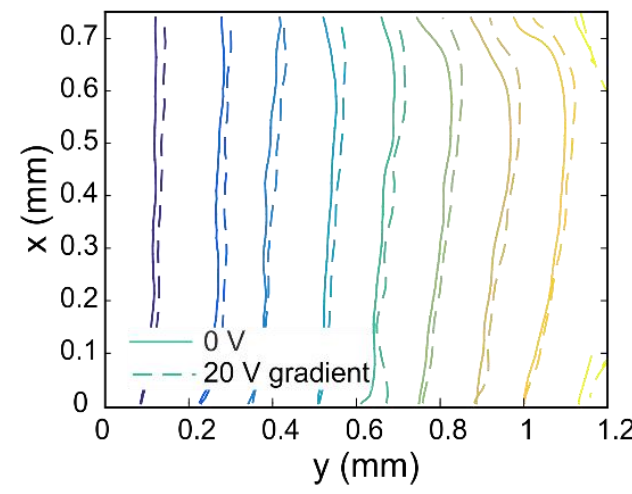

(b) $20 \mathrm{~V}$ gradient

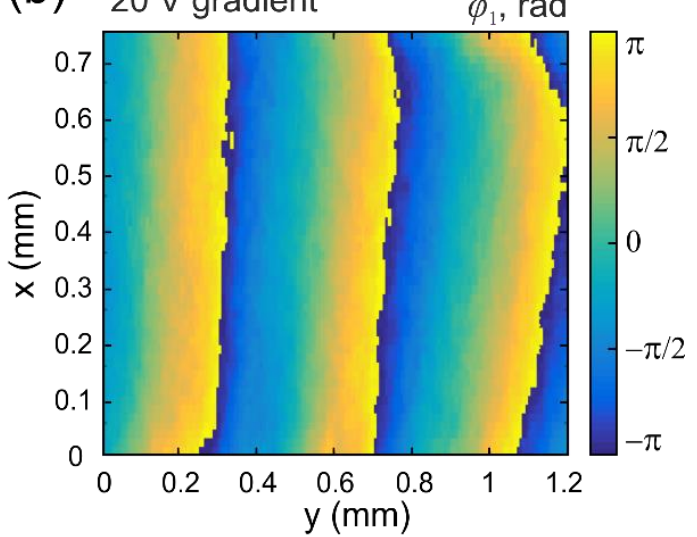

(d)

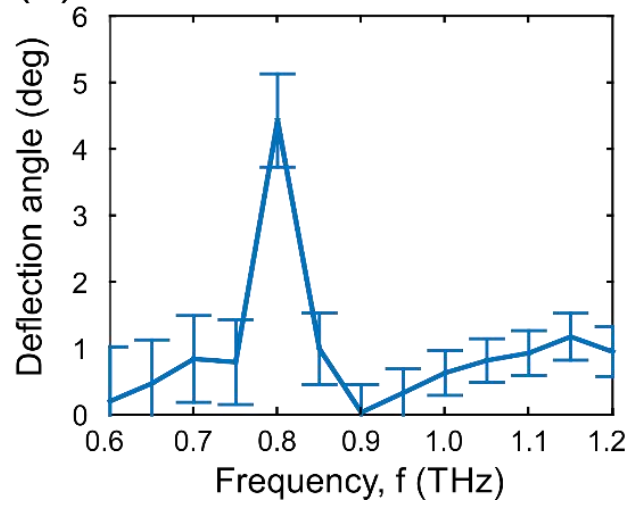

Figure 3. (a) An image of the wavefronts transmitted by the spatial phase modulator at $0.8 \mathrm{THz}$ in the off state plotted in terms of an experimentally mapped electromagnetic phase. (b) Same as (a) but plotted for the case when a $20 \mathrm{~V}$ linear gradient was applied to the modulator along the $x$-axis. (c) Experimentally measured contours of the wavefronts transmitted by the spatial phase modulator at $0.8 \mathrm{THz}$ with (dashed lines) and without (solid lines) the voltage gradient applied. The contours are separated by a phase difference of $2 \mathrm{rad}$. (d) Measured beam deflection produced by the spatial phase modulator due to $20 \mathrm{~V}$ gradient as a function of frequency.

Furthermore, the measured peak value of the wavefront deflection angle falls short of the theoretical limit by only $10 \%$. Minor deviations between the modelled and experimental results are attributed to the fact that the fabricated SPM was driven by a linear gradient of voltage, which could not generate a truly linear gradient for the tilt angle of LC molecules, $\theta$, as was assumed in our numerical model. 


\section{CONCLUSION}

We have presented the design of a novel ultra-thin large-area terahertz spatial phase modulator for wavefront correction and experimentally demonstrated its operation at $0.8 \mathrm{THz}$. Our SPM is based on a spatially addressable stack of two specially designed metallic metasurfaces integrated with an LC cell similar in thickness to conventional optical LC cells (as employed by many optical devices). With the thickness of the LC layer of only $12 \mu \mathrm{m}(\sim 0.03 \lambda)$ the demonstrated SPM is capable of changing the phase of transmitted terahertz wavefronts by more than $30^{\circ}(\sim 0.1 \lambda)$ with a spatial resolution of $85 \mu \mathrm{m}$ (better than $0.23 \lambda$ ), and requires the driving voltage of as low as $20 \mathrm{~V}$. In the proof-of-concept demonstration the SPM was programmed to tilt transmitted wavefronts by applying a linear voltage gradient across $0.72 \mathrm{~mm}^{2}\left(\sim 5 \lambda^{2}\right)$ large active area of the device. The wavefront deflection angle of 4.5 degrees, calculated based on the experimental data agreed very well with the value obtained in the course of numerical simulations $\left(5^{\circ}\right)$, indicating on the exceptional robustness of our SPM concept. While our demonstration involved configuring the SPM with a simple phase gradient profile, the design of the SPM allows 'imprinting' 2D phase profile of any spatial complexity with sub-wavelength resolution in a manner similar to the passively addressable scheme of LC displays. Importantly, the manufacturing of the proposed SPM largely involves the well-established LC-device technology and high-resolution photolithography technique, and requires inexpensive, commercially available substrates materials, such as quartz and silicon.

\section{ASSOCIATED CONTENT}

\section{Supporting Information}

The Supporting Information is available free of charge via Internet at https://pubs.acs.org/ Additional data on the phase of a directly transmitted THz wave of the spatial phase modulator; a photograph of the assembled THz spatial phase modulator; a schematic of the THz-field imaging setup; Calculation of the tilt angle for beams with curved wavefronts. 


\section{ACKNOWLEDGEMENTS}

This work is supported by the the Royal Society through grant IE130472 and by the UK's Engineering and Physical Sciences Research Council (EPSRC) through an extension of Career Acceleration Fellowship EP/G00515X/1.

\section{REFERENCES}

[1] I.-C. Khoo, Liquid Crystals 2nd Ed., Wiley, NY, USA 2007.

[2] G. D. Love. Wave-front Correction and Production of Zernike Modes with a Liquid-crystal Spatial Light Modulator. Appl. Opt. 1997, 36, 1517.

[3] S. R. Restaino, S.W. Teare. Introduction to Liquid Crystals for Optical Design and Engineering, SPIE press, Bellingham, WA, USA 2015. https://doi.org/10.1117/3.2197135

[4] A. S. Ostrovsky, C. Rickenstorff-Parrao, V.Arrizón. Generation of the "Perfect" Optical Vortex using a Liquid-crystal Spatial Light Modulator. Opt. Lett. 2013, 38, 534.

[5] L. Hu, L. Xuan, Yo. Liu, Zh. Cao, D. Li, Qu. Mu. Phase-only Liquid-crystal Spatial Light Modulator for Wave-front Correction with High Precision. Opt. Express 2004, 12, 6403.

[6] M. A. Olvera Santamaría, J. L. Rodríguez Garciapiña, J. García García, A. S. Ostrovsky. Generation of a Partially Coherent Secondary Source with Bessel-mode Vortex Structure by liquid crystal Spatial Light Modulator. Opt. Commun. 2019, 439, 312.

[7] M. Tonouchi. Cutting-edge Terahertz Technology. Nature Photon. 2007, 1, 97.

[8] N. J. Laurita, B. Cheng, R. Barkhouser, V. A. Neumann, and N. P. Armitage. A Modified 8f Geometry with Reduced Optical Aberrations for Improved Time Domain Terahertz Spectroscopy. J. Infrared Millim. Terahertz Waves 2016, 37, 894.

[9] M. Brossard, J.-F. Sauvage, M. Perrin, E. Abraham. Terahertz Adaptive Optics with a Deformable Mirror. Opt. Lett. 2018, 43, 1594.

[10] X. Lin, J. Wu, W. Hu, Z. Zheng, Z. Wu, G. Zhu, F. Xu, B. Jin, Y. Lu. Self-polarizing Terahertz Liquid Crystal Phase Shifter. AIP Adv. 2011, 1, 032133. 
[11] F. Fan, Y. Hou, Z.-W. Jiang, X.-H. Wang, S.-J. Chang. Terahertz Modulator Based on Insulator-metal Transition in Photonic Crystal Waveguide. Appl. Opt. 2012, 51, 4589.

[12] F. Fan, Y. Liu, J. Li, X.-H. Wang, S.-J. Chang. Active Terahertz Directional Coupler based on Phase Transition Photonic Crystals. Opt. Commun. 2015, 336, 59.

[13] B. Sensale-Rodriguez, R. Yan, M.M. Kelly, T. Fang, K. Tahy, W.S. Hwang, D. Jena, L. Liu, H.G. Xing. Broadband Graphene Terahertz Modulators enabled by Intraband Transitions. Nat. Commun. 2012, 3, 780.

[14] W. Gao, J. Shu, K. Reichel, D.V. Nickel, X. He, G. Shi, R. Vajtai, P.M. Ajayan, J. Kono, D.M. Mittleman. High-Contrast Terahertz Wave Modulation by Gated Graphene Enhanced by Extraordinary Transmission through Ring Apertures. Nano Lett. 2014, 14, 1242.

[15] R. Kersting, G. Strasser, K. Unterrainer. Terahertz Phase Modulator. Electron. Lett. 2000, $36,1156$.

[16] I. H. Libon, S. Baumgärtner, M. Hempel, N. E. Hecker, J. Feldmann, M. Koch, P. Dawson. An Optically Controllable Terahertz Filter. Appl. Phys. Lett. 2000, 76, 2821.

[17] C. Chen, Y. Zhu, Y. Zhao, J.H. Lee, H. Wang, A. Bernussi, M. Holtz, Z. Fan. VO Multidomain Heteroepitaxial Growth and Terahertz Transmission Modulation. Appl. Phys. Lett. 2010, 97, 211905.

[18] V. G. Veselago, E. E. Narimanov. The Left Hand of Brightness: Past, Present and Future of Negative Index Materials. Nat. Mater. 2006, 5, 759.

[19] H.T. Chen, J.F. O'Hara, A.K. Azad, A.J. Taylor. Manipulation of Terahertz Radiation using Metamaterials. Laser Photonics Rev. 2011, 5, 513.

[20] M. Liu, Q. Yang, A. A. Rifat, V. Raj, A. Komar, J. Han, M. Rahmani, H. T. Hattori, D. Neshev, D. A. Powell, I. V. Shadrivov. Deeply Subwavelength Metasurface Resonators for Terahertz Wavefront Manipulation. Adv. Optical Mater. 2019, 7, 1900736. 
[21] R. Kowerdziej, L. Jaroszewicz, M. Olifierczuk, Ja. Parka. Experimental Study on Terahertz Metamaterial Embedded in Nematic Liquid Crystal. Appl. Phys. Lett. 2015, 106, 092905.

[22] C. Kadlec, V. Skoromets, F. Kadlec, H. Němec, H.-T. Chen, V. Jurka, K. Hruška, P. Kužel. Electric-field Tuning of a Planar Terahertz Metamaterial Based on Strained $\mathrm{SrTiO}_{3}$ Layers. J. Phys. D: Appl. Phys. 2018, 51, 054001.

[23] Y. -G. Jeong, Y.-M. Bahk, D. -S. Kim. Dynamic Terahertz Plasmonics Enabled by Phase Change Materials. Adv. Optical Mater. 2020, 8, 1900548.

[24] Y. Sanari, T. Tachizaki, Y. Saito, K. Makino, P. Fons, A. V. Kolobov, J. Tominaga, K. Tanaka, Y. Kanemitsu, M. Hase, H. Hirori. Zener Tunneling Breakdown in Phase-Change Materials Revealed by Intense Terahertz Pulses. Phys. Rev. Lett. 2018, 121, 165702.

[25] P. Pitchappa, A. Kumar, S. Prakash, H. Jani, T. Venkatesan, R. Singh. Chalcogenide Phase Change Material for Active Terahertz Photonics. Adv. Mater. 2019, 31, 1808157.

[26] S. Wang, L. Kang, D. H. Werner. Active Terahertz Chiral Metamaterials Based on Phase Transition of Vanadium Dioxide $\left(\mathrm{VO}_{2}\right)$. Sci. Rep. 2018, 8, 189.

[27] Y.-G. Jeong, S. Han, J. Rhie, J.-S. Kyoung, J.-W. Choi, N. Park, S. Hong, B.-J. Kim, H.-T. Kim, D.-S. Kim. A Vanadium Dioxide Metamaterial Disengaged from Insulator-to-Metal Transition. Nano Lett. 2015, 15, 6318.

[28] H.-T. Chen, W. J. Padilla, M. J. Cich, A. K. Azad, R. D. Averitt, A. J. Taylor. A Metamaterial Solid-state Terahertz Phase Modulator. Nat. Photonics 2009, 3, 148.

[29] W.L. Chan, H.-T. Chen, A.J. Taylor, I. Brener, M.J. Cich, D.M. Mittleman. A Spatial Light Modulator for Terahertz Beams. Appl. Phys. Lett. 2009, 94, 213511.

[30] M. Liu, M. Susli, D. Silva, G. Putrino, H. Kala, Sh. Fan, M. Cole, L. Faraone, V. P. Wallace, W. Padilla, D. Powell, I. Shadrivov, M. Martyniuk. Ultrathin Tunable Terahertz Absorber based on MEMS-driven Metamaterial. Microsyst. Nanoeng. 2017, 3, 17033. 
[31] M. Jiang, F. Hu, Y. Qian, L. Zhang, W. Zhang, J. Han. Tunable Terahertz Band-pass Filter based on MEMS Reconfigurable Metamaterials. J. Phys. D: Appl. Phys. 2020, 53, 065107.

[32] S.H. Lee, M. Choi, T.-T. Kim, S. Lee, M. Liu, X. Yin, H.K. Choi, S.S. Lee, C.-G. Choi, S.Y. Choi, X. Zhang, B. Min. Switching terahertz waves with gate-controlled active graphene metamaterials. Nat. Mater. 2012, 11, 936.

[33] T. Low, P. Avouris. Graphene Plasmonics for Terahertz to Mid-Infrared Applications. ACS Nano 2014, 8, 1086.

[34] O. Buchnev, J. Y. Ou, M. Kaczmarek, N. I. Zheludev, V. A. Fedotov. Electro-optical Control in a Plasmonic Metamaterial Hybridised with a Liquid-crystal Cell. Opt. Express 2013, 21, 1633.

[35] O. Buchnev, N. Podoliak, V. A. Fedotov. Liquid Crystal-filled Meta-pixel with Switchable Asymmetric Reflectance and Transmittance. J. Mol. Liq. 2018, 267, 411.

[36] P. A. Kossyrev, A. Yin, S. G. Cloutier, D. A. Cardimona, D. Huang, P. M. Alsing, J. M. Xu. Electric Field Tuning of Plasmonic Response of Nanodot Array in Liquid Crystal Matrix. Nano Lett. 2005, 5, 1978.

[37] M. V. Gorkunov, I. V. Kasyanova, V. V. Artemov, M. I. Barnik, A. R. Geivandov, S. P. Palto. Fast Surface-Plasmon-Mediated Electro-Optics of a Liquid Crystal on a Metal Grating. Phys. Rev. Applied 2017, 8, 054051.

[38] M. Decker, Ch. Kremers, A. Minovich, I. Staude, A. E. Miroshnichenko, D. Chigrin, D. N. Neshev, Ch. Jagadish, Yu. S. Kivshar. Electro-optical Switching by Liquid-Crystal Controlled Metasurfaces. Opt. Express 2013, 21, 8879.

[39] O. Buchnev, N. Podoliak, M. Kaczmarek , N. I. Zheludev, V. A. Fedotov. Electrically Controlled Nanostructured Metasurface Loaded with Liquid Crystal: toward Multifunctional Photonic Switch. Adv. Opt. Mater. 2015, 3, 674. 
[40] Sh.-Q. Li, X. Xu, R. Veetil, V. Valuckas, R. Paniagua-Domnguez, A. I. Kuznetsov. Phaseonly transmissive spatial light modulator based on tunable dielectric metasurface. Science 2019, $364,1087$.

[41] J. Wu, Z. Shen, Sh. Ge, B. Chen, Zh. Shen, T. Wang, C. Zhang, W. Hu, K. Fan, W. Padilla, Ya. Lu, B. Jin, J. Chen, P. Wu. Liquid Crystal Programmable Metasurface for Terahertz Beam Steering. Appl. Phys. Lett. 2020, 116, 131104.

[42] O. Buchnev, J. Wallauer, M. Walther, M. Kaczmarek, N. I. Zheludev, V. A. Fedotov. Controlling Intensity and Phase of Terahertz Radiation with an Optically Thin Liquid CrystalLoaded Metamaterial. Appl. Phys. Lett. 2013, 103, 141904.

[43] N. Chikhi, M. Lisitskiy, G. Papari, V. Tkachenko, A. Andreone. A Hybrid Tunable THz Metadevice Using a High Birefringence Liquid Crystal. Sci. Rep. 2016, 6, 34536.

[44] COMSOL Multiphysics, version 5.5; software for technical computation; COMSOL Ltd.: Cambridge, UK 2019.

[45] C. L. Davies, J. B. Patel, C. Q. Xia, L. M. Herz, M. B. Johnston. Temperature-Dependent Refractive Index of Quartz at Terahertz Frequencies. J. Infrared Millim. Terahertz Waves 2018, $39,1236$.

[46] U. Chodorowa, Ja. Parka, K. Garbat, N. Pałka, K. Czuprynski. Spectral Investigation of Nematic Liquid Crystals with High Optical Anisotropy at THz Frequency Range. Phase Transit. 2012, 85, 337.

[47] V. A. Fedotov, P. L. Mladyonov, S. L. Prosvirnin, N. I. Zheludev. Planar Electromagnetic Metamaterial with a Fish Scale Structure. Phys. Rev. E 2005, 72, 056613.

[48] Ja. Herman, Pr. Kula. Design of New Super-high Birefringent Isothiocyanato Bistolanes Synthesis and Properties. Liq. Cryst. 2017, 44, 1462. 
[49] R. Kowerdziej, Ja. Parka, J. Krupka, M. Olifierczuk, E. Nowinowski-Kruszelnicki, L. Jaroszewicz, O. Chojnowska. Dielectric Properties of Highly Anisotropic Nematic Liquid Crystals for Tunable Microwave Components. Appl. Phys. Lett. 2013, 103, 172902.

[50] B. Luk'yanchuk, N. I. Zheludev, S. A. Maier, N. J. Halas, P. Nordlander, H. Giessen, and C. T. Chong. The Fano Resonance in Plasmonic Nanostructures and Metamaterials. Nat. Mater. 2010, 9, 707.

[51] Y. Utsumi, T. Kamei, R. Naito, K. Saito. Measurement Methods of Nematic Liquid Crystal Response Time. Mol. Cryst. Liq. Cryst. 2005, 434, 337.

[52] N. Podoliak, O. Buchnev, M. Herrington, E. Mavrona, M. Kaczmarek, A. G. Kanaras, E. Stratakis, J.-F. Blach, J.-F. Henninot, M. Warenghem. Elastic constants, viscosity and response time in nematic liquid crystals doped with ferroelectric nanoparticles. RSC Advances 2014, 4, 46068.

[53] D. C. Zografopoulos, R. Beccherelli. Tunable Terahertz Fishnet Metamaterials based on Thin Nematic Liquid Crystal Layers for Fast Switching. Sci. Rep. 2015, 5, 13137.

[54] R. Kowerdziej, J. Wróbel, P. Kula. Ultrafast Electrical Switching of Nanostructured Metadevice with Dual-frequency Liquid Crystal. Sci. Rep. 2019, 9, 20367.

[55] A. Bitzer, H. Merbold, A. Thoman, T. Feurer, H. Helm, M. Walther. Terahertz Near-field Imaging of Electric and Magnetic Resonances of a Planar Metamaterial. Opt. Express 2009, 17, 3826. 


\section{For Table of Contents Use Only}

\section{Metasurface-based Optical Liquid Crystal Cell as an Ultra-Thin Spatial Phase Modulator for THz Applications}

O. Buchnev, N. Podoliak, K. Kaltenecker, M. Walther, and V. A. Fedotov

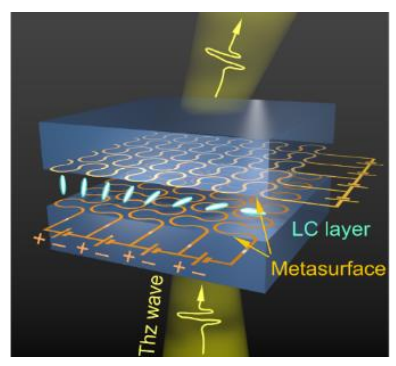

Ultra-thin spatial phase modulator for correcting terahertz wavefronts with sub-wavelength spatial resolution is developed as a combination of spatially addressable resonant planar metamaterials and an optically thin $(<0.04 \lambda)$ low-voltage $(<20 \mathrm{~V})$ liquid crystal cell. The developed modulator allows 'imprinting' $2 \mathrm{D}$ phase profile of any spatial complexity with a spatial resolution better than $0.23 \lambda$. 